\title{
Retuning PI controller to improve the control performance in CO2 removal process, subang field
}

\author{
Abdul Wahid ${ }^{1, *}$, and Mahdi ${ }^{1}$ \\ ${ }^{1}$ Chemical Engineering Department, Universitas Indonesia, Depok, Indonesia
}

\begin{abstract}
A proportional - integral controller retuning is performed on $\mathrm{CO}_{2}$ removal process, Subang field. Retuning is carried out to improve the control performance on the process. The method used is to evaluate the controllers that are retuned the controller parameters, identify the system using the first-order plus dead time model (FOPDT), tune the controller parameters using the Ziegler-Nichols, Wahid-Rudi-Victor (WRV), Cohen-Coon, autotuning, and fine tuning, and the last, the control performance tests using set point (SP) tracking and disturbance rejection with performance indicator is integral of square error (ISE). The result is that there are three controllers that are retuning the control parameters, i.e., the feed gas pressure control (PIC - 1101), the makeup water flow control (FIC - 1102), and the amine circulation flow control (FIC - 1103). Only a fine tuning method produces the best control performance compared to previous settings in the field, with performance improvements of 77,42\% (PIC - 1101), 90,59\% (FIC - 1102), and 13,06\% (FIC - 1103) for $-5 \%$ set point (SP) tracking. While for disturbance rejection, fine tuning gives performance improvements of $86,04 \%$ (PIC - 1101), 90,8\% (FIC - 1102), and 24,8\% (FIC - 1103). Thus, retuning PI controllers work well.
\end{abstract}

\section{Introduction}

Natural gas should be processed before it can be used. One such process is the removal of acid gas content (sweetening), i.e. $\mathrm{CO}_{2}$ and $\mathrm{H}_{2} \mathrm{~S}$. One of the industries that conduct $\mathrm{CO}_{2}$ removal process from natural gas is PT $\mathrm{X}$ Subang field, where the content of $\mathrm{H}_{2} \mathrm{~S}$ in the feed gas is negligible. The type of controller used in PT $\mathrm{X}$ is proportional - integral controller. Over time, the performance of a plant will decrease, causing the old controller tuning no longer appropriate to be used, including on PT X Subang field, hence controller performance is decreased. Objective of this study is to improve controller performance by changing the tuning to a better one obtained by retuning. Therefore, it is necessary to do a controller retuning to improve the performance of the controller at $\mathrm{CO}_{2}$ removal plant PT X Subang field, so stability will be achieved faster when there is a disturbance or set point (SP) changes.

Veronesi and Visioli [1-2] have conducted a proportional-integral (PI) and proportional-integralderivative (PID) controller retuning for set point (SP) tracking and disturbance rejection, and it proved that controller performance could be improved by retuning. Both [3-4], have also apply it on temperature control in energy production plant from renewable sources, in particular by using palm oil as a fuel, and other processes. The model used for retuning is based on a closed loop model testing, and results on an FOPDT model. In this study, an open loop model testing will be conducted with the same model result, i.e. FOPDT, while the retuning method used will be searched for the correct method. Open loop model testing is used because this study intends to obtain empirical model of the process, then used it to do the retuning using some process empirical model based methods.

\section{Methodology}

\subsection{Field Data Collection}

A plant visit will be undertaken to collect data required for the research, including P\&ID, daily process data, valve sizing, and controller data. PFD of the plant [5] is shown in Figure 1.

This Subang field $\mathrm{CO}_{2}$ removal plant consists of 2 trains, designed to reduce $\mathrm{CO}_{2}$ content in feed gas from 23 mole $\%$ to less than $5 \mathrm{~mole} \%$. The solvent used is aMDEA with piperazine as its activator, and the designed capacity of each train is 100 MMSCFD. However, this plant can only operate with the capacity of 60 - 70 MMSCFD for each train nowadays.

\subsection{Simulation Preparation}

This research is carried out with the help of a process simulator, and will run on the dynamic mode of the simulator. Before the dynamic simulation is made, the simulation will be made on steady state mode first, until the simulation describes the real condition in the field.

\footnotetext{
*Corresponding author: wahid@che.ui.ac.id
} 


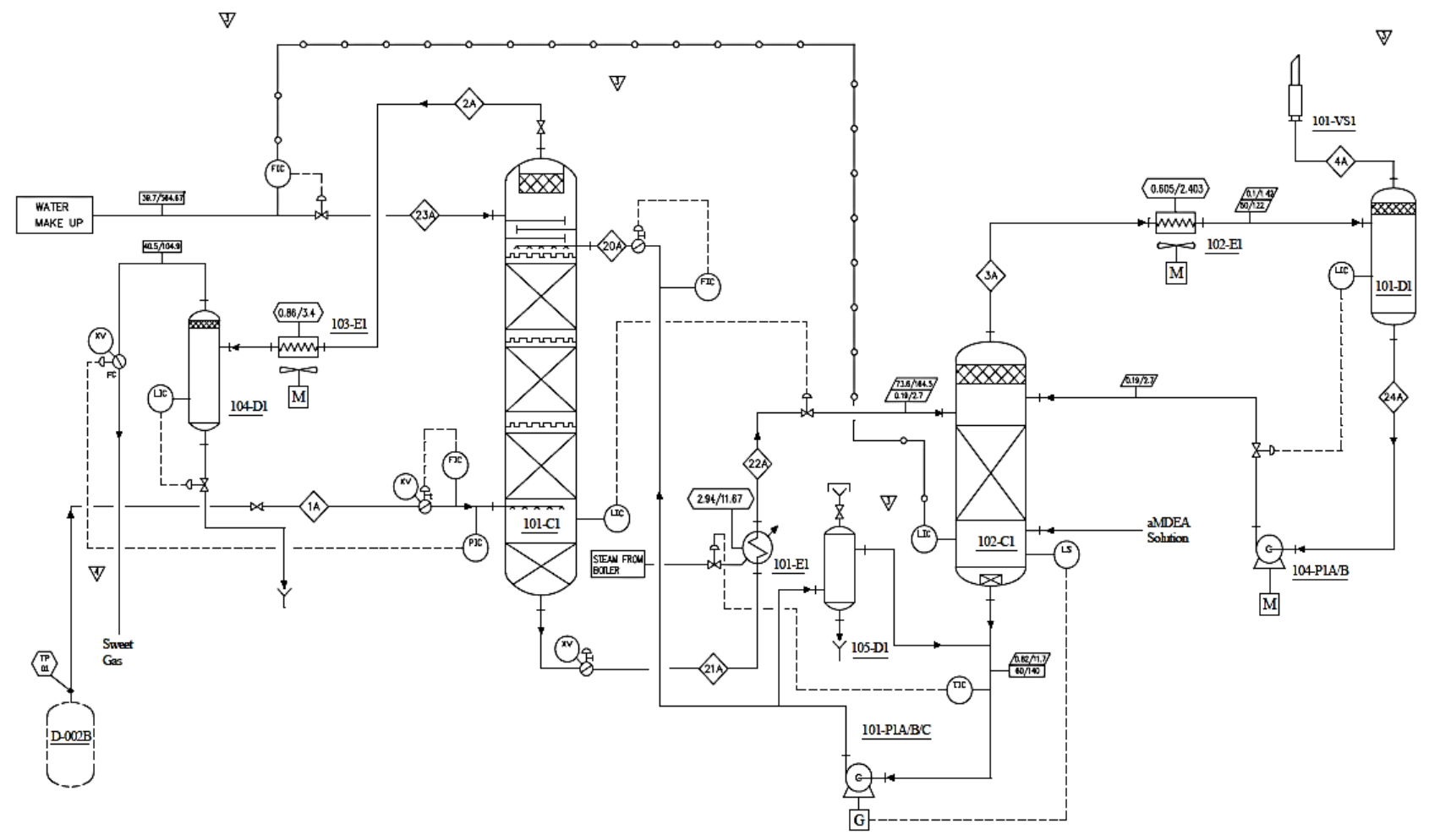

Fig. 1. PFD of $\mathrm{CO}_{2}$ Removal Plant Subang Field

After the steady state simulation is completed and the result matches the field condition, then valve and PI controller will be added according to their placement on the field. Then, the installed valve will be sized first by entering the valve size from the field, so the simulation of controller retuning performed describes the actual condition.

After the steady state simulation, valve, and controllers are ready, then the simulation will be switched to dynamic mode. In dynamic mode, the simulation will run until stable condition is obtained, so then the research can continue. However, before the simulation runs, the autotuner feature will be used to provide initial tuning for the controllers, so that stability could be achieved faster.

Configuration for simulation of the plant can be seen in Figure 2, where this study focused only on PIC - 1101, FIC - 1102 and FIC - 1103 controller, which has been circled in red on Figure 2. PIC - 1101 controls the absorber inlet gas pressure from the well, FIC - 1102 controls makeup water flow rate to absorber, and FIC 1103 controls the flow rate of amine circulation to absorber column.

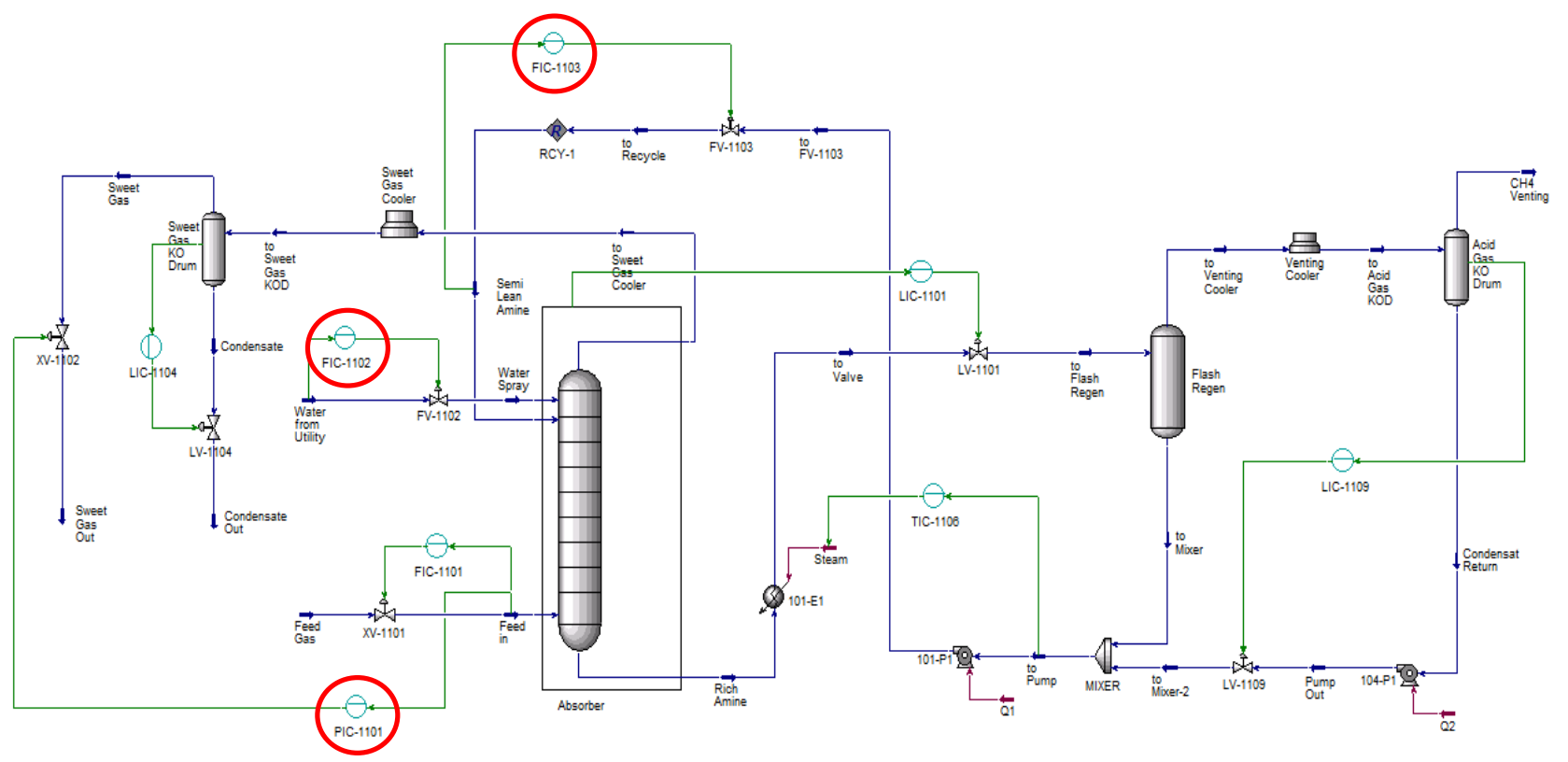

Fig. 2. Dynamic Simulation of CO2 Removal Process Subang Field 


\subsection{PRC and Empirical Models}

To determine the empirical models for tuning equations, Process Reaction Curve (PRC) is made by increasing 5\% of valve opening (OP), usually called model testing. PRC is the response curve of process variable (PV) caused by changes of valve opening.

Process empirical modeling will be conducted after the PRC is obtained, and the method used is FOPDT. FOPDT is an empirical modeling method to obtain the process empirical model [6]. The transfer function of FOPDT [6] is shown by equation (1).

$$
G_{p}(s)=\frac{K_{p} e^{-\theta s}}{\tau s+1}
$$

where, $K_{P}$ is process gain describing the distance of PV move; $\tau$ is time constant describing PV responds time; and $\theta$ is dead time describing the delay occurs before the $\mathrm{PV}$ begins to move.

The process empirical model, $\mathrm{Kp}, \tau$, and $\theta$, will be determined with method II FOPDT [7] in equation (2), (3), and (4).

$$
K p=\frac{\Delta}{\delta}
$$

where, $\Delta$ is the change of PV; and $\delta$ is the change of OP.

$$
\tau=1.5\left(t_{63 \%}-t_{28 \%}\right)
$$

where, $t$ is the time needed for PV to reach $28 \%$ and $63 \%$ of the final stable value.

$$
\theta=t_{63 \%}-\tau-t_{0}
$$

where, $t_{0}$ is the time when model testing starts.

\subsection{Controller Retuning}

The methods used for this study are Ziegler - Nichols (PRC), Wahid - Rudi - Victor (WRV), Cohen - Coon (CC), simulator autotuner, and fine tuning. The equation for PI controller retuning from ZN (PRC) [6], WRV [8], and $C C[6]$ are shown in Table 1. Fine tuning method is conducted by refining the best tuning from $\mathrm{ZN}, \mathrm{WRV}$, or $\mathrm{CC}$ to a better tuning.

Table 1. Tuning equation for ZN (PRC), WRV, and CC

\begin{tabular}{|c|c|c|}
\hline Method & Controller Gain, Kc & Integral Time, Ti \\
\hline $\begin{array}{c}\text { ZN } \\
\text { (PRC) }\end{array}$ & $\frac{0.9}{K}\left(\frac{\theta}{\tau}\right)^{-1}$ & $3.33 \theta$ \\
\hline WRV & $\begin{array}{l}K_{c} \\
=\frac{0.0433 \tau+0.8353}{K}\end{array}$ & $\begin{array}{l}\tau_{i} \\
=1.027 \tau+10.777\end{array}$ \\
\hline CC & $\frac{1}{K}\left(\frac{\theta}{\tau}\right)^{-1}\left(0.9+\frac{\theta}{12 \tau}\right)$ & $\frac{\theta(30+3(\theta / \tau))}{9+20(\theta / \tau)}$ \\
\hline
\end{tabular}

\subsection{Controller Performance}

All controllers tuning performance is tested by doing setpoint tracking and disturbance rejection. SP Tracking is conducted by reducing 5\% SP for FIC - 1102 and FIC 1103, while reducing SP to $509 \mathrm{psig}$ for PIC -1101 . The disturbance given for the controller is 20 MMSCFD reduction of inlet feed gas flow rate.
Parameter used for controller performance in this study is integral of square error (ISE) [6] shown on equation (5). After all tuning had been tested, ISE of each and every one of the tuning will be compared. Tuning with the lowest ISE value is the best tuning.

$$
I S E=\int(S P(t)-C V(t))^{2} d t=\int(E(t))^{2} d t
$$

where, SP is set point describing the value set for the variable; $\mathrm{CV}$ is controlled variable describing the current variable value; and $\mathrm{E}$ is error describing the value difference of SP and CV. All of those parameters are in the function of time.

\section{Results and discussion}

\subsection{PRC and FOPDT Models}

After performing model testing for every controller, PRCs are obtained for each of them. PRC of PIC - 1101, FIC 1102, and FIC - 1103 are shown in Figure 3.

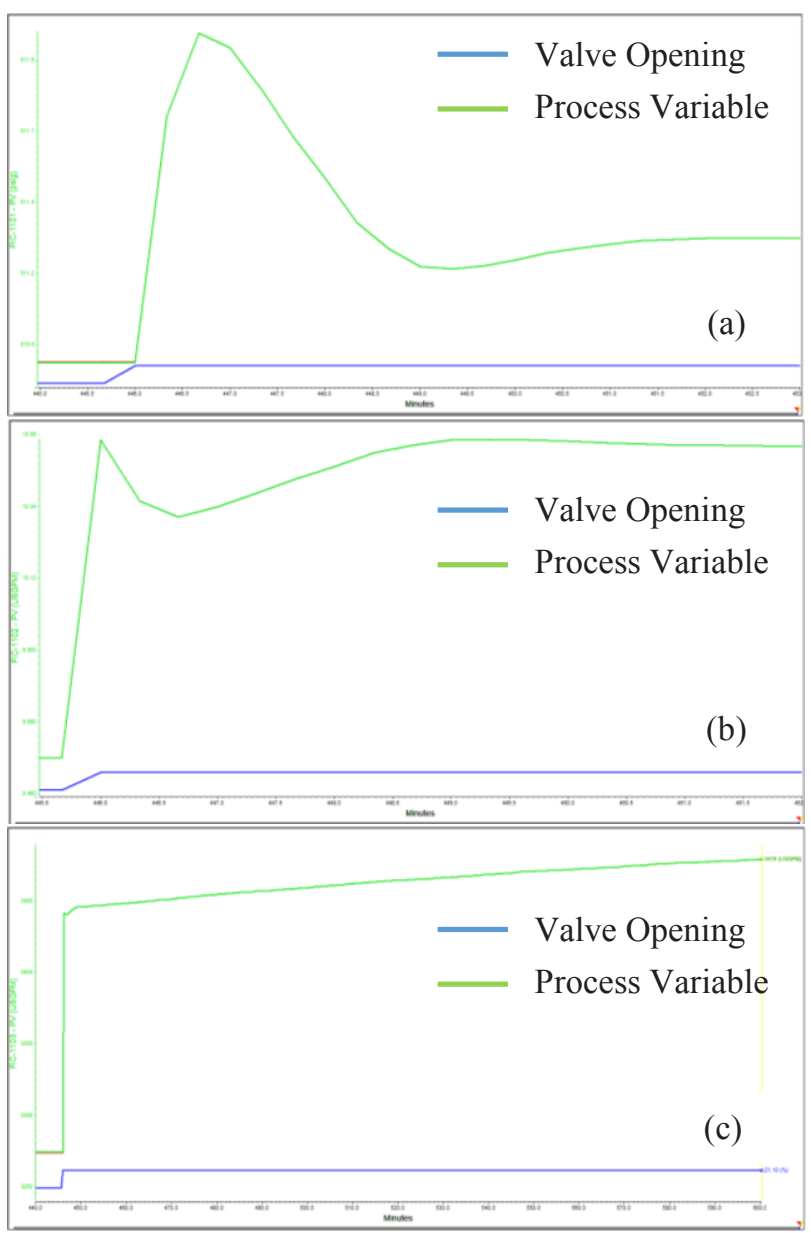

Fig. 3. PRC results for model testing: (a) PIC -1101 , (b) FIC 1102, (c) FIC -1103

From the PRCs, process empirical models based on FOPDT are obtained and shown in Table 2. 
Table 2. FOPDT Models

\begin{tabular}{|c|c|c|c|}
\hline Controller & Kp & $\boldsymbol{\tau}$ & $\boldsymbol{\theta}$ \\
\hline PIC -1101 & 0.09 & 0.09 & 0.02 \\
\hline FIC -1102 & 0.19 & 0.19 & 0.03 \\
\hline FIC -1103 & 41.04 & 0.21 & 0.04 \\
\hline
\end{tabular}

\subsection{Tuning Value}

Result of the test shows that the best tuning among $\mathrm{ZN}$, $\mathrm{WRV}$, and CC is tuning with WRV method, hence fine tuning is conducted based on tuning value from WRV method and refined to get a better tuning. Tuning values for every method are shown in Table 3.

Table 3. Controller Tuning

\begin{tabular}{|c|c|c|c|c|}
\hline \multirow{2}{*}{$\begin{array}{c}\text { Tuning } \\
\text { Method }\end{array}$} & \multirow{2}{*}{ Tuning } & \multicolumn{3}{|c|}{ Controller } \\
\cline { 3 - 5 } & & $\begin{array}{c}\text { PIC } \\
\mathbf{1 1 0 1}\end{array}$ & $\begin{array}{c}\text { FIC } \\
\mathbf{1 1 0 2}\end{array}$ & $\begin{array}{c}\text { FIC } \\
\mathbf{1 1 0 3}\end{array}$ \\
\hline \multirow{2}{*}{ Field } & $\mathrm{Kc}$ & 7.2 & 0.5 & 0.37 \\
\cline { 2 - 5 } & $\mathrm{Ti}(\mathrm{s})$ & 46.1 & 10 & 10.6 \\
\hline \multirow{2}{*}{$\begin{array}{c}\text { ZN } \\
\text { (PRC) }\end{array}$} & $\mathrm{Kc}$ & 49.44 & 33.24 & 0.11 \\
\cline { 2 - 5 } & $\mathrm{Ti}(\mathrm{s})$ & 0.06 & 0.09 & 0.14 \\
\hline \multirow{2}{*}{ WRV } & $\mathrm{Kc}$ & 9.23 & 4.4 & 0.02 \\
\cline { 2 - 5 } & $\mathrm{Ti}(\mathrm{s})$ & 10.87 & 10.98 & 11 \\
\hline \multirow{2}{*}{ CC } & $\mathrm{Kc}$ & 50.36 & 33.68 & 0.11 \\
\cline { 2 - 5 } & $\mathrm{Ti}(\mathrm{s})$ & 0.04 & 0.07 & 0.1 \\
\hline \multirow{2}{*}{$\begin{array}{c}\text { Auto } \\
\text { tuner }\end{array}$} & $\mathrm{Kc}$ & 16.9 & 8.44 & 0.25 \\
\cline { 2 - 5 } & $\mathrm{Ti}(\mathrm{s})$ & 2.93 & 0.733 & 0.733 \\
\hline \multirow{2}{*}{$\begin{array}{c}\text { Fine } \\
\text { Tuning }\end{array}$} & $\mathrm{Kc}$ & 10 & 4 & 0.3 \\
\cline { 2 - 5 } & $\mathrm{Ti}(\mathrm{s})$ & 0.183 & 0.183 & 0.183 \\
\hline
\end{tabular}

\subsection{SP tracking}

Result of controller response for set point tracking shows that $\mathrm{ZN}$ (PRC), WRV, and CC tuning method is worse than current tuning, so they're not displayed on the graph. Controller responses for set point tracking with the tunings observed are shown in Figure 4 with controlled variable $(\mathrm{CV})$ on the $\mathrm{y}$ axis, and time in seconds on the $\mathrm{x}$ axis.

Figure 4 shows that the tuning which can set the controlled variable back to set point fastest when the set point is decreased is fine tuning. For 5\% set point (SP) tracking, retuning with fine tuning gives $77.42 \%$ improvement for PIC - 1101, 90.59\% for FIC - 1102, and $13.06 \%$ for FIC -1103 . ISE value of every tuning in every controller for SP tracking is shown in Table 4.

Table 4. ISE Value for SP Tracking

\begin{tabular}{|c|c|c|c|}
\hline Controllers & Fine Tuning & Field & Autotuner \\
\hline PIC-1101 & 4.64 & 20.55 & 31.41 \\
\hline FIC-1102 & 0.27 & 2.87 & 0.45 \\
\hline FIC-1103 & 3783 & 4352 & 73477 \\
\hline
\end{tabular}
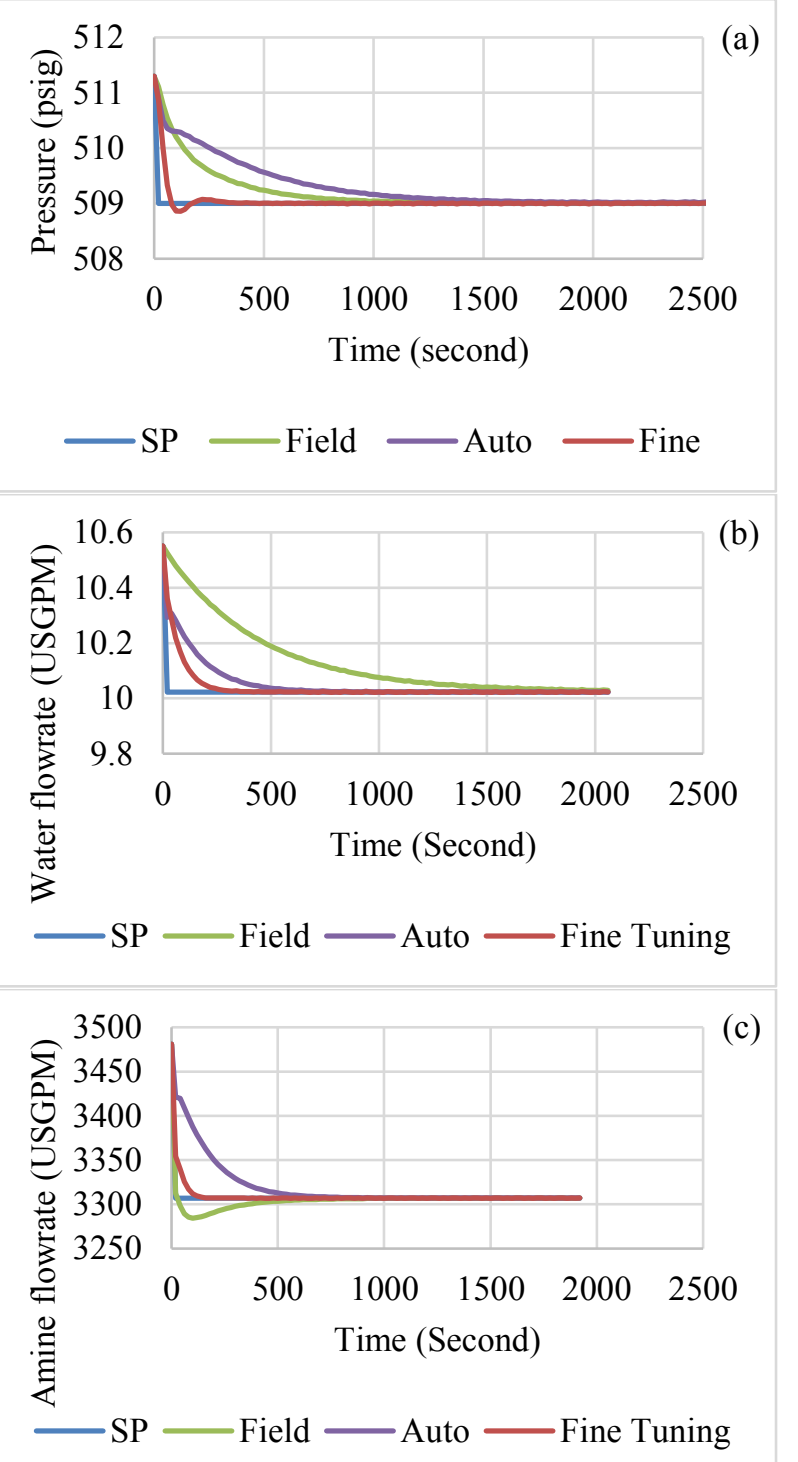

Fig. 4. SP Tracking Results (-5\%): (a) PIC - 1101, (b) FIC 1102, (c) FIC - 1103

\subsection{Disturbance rejection}

Result of controller response for disturbance rejection also shows that ZN (PRC), WRV, and CC tuning method are worse than current tuning, so they're not displayed on the graph neither. Controller responses for disturbance rejection with the tunings observed are shown in Figure 5 with controlled variable $(\mathrm{CV})$ on the $\mathrm{y}$ axis, and time in seconds on the $\mathrm{x}$ axis.

Figure 5 shows that the tuning which can set the controlled variable back to set point fastest when there is a 20 MMSCFD decrease in feed gas flow rate is fine tuning. For disturbance rejection, fine tuning is also the best method, it gives $86.04 \%$ improvement for PIC $1101,90.8 \%$ for FIC - 1102, and $24.8 \%$ for FIC - 1103 . ISE value of every tuning in every controller for disturbance rejection is shown in Table 5 . 


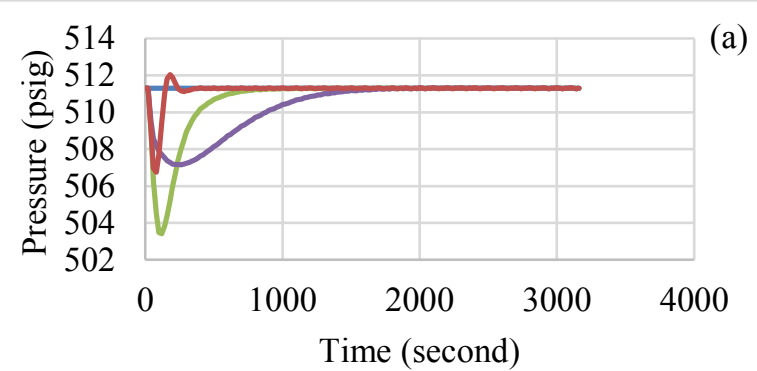

$\longrightarrow$ SP Field —Auto —Fine
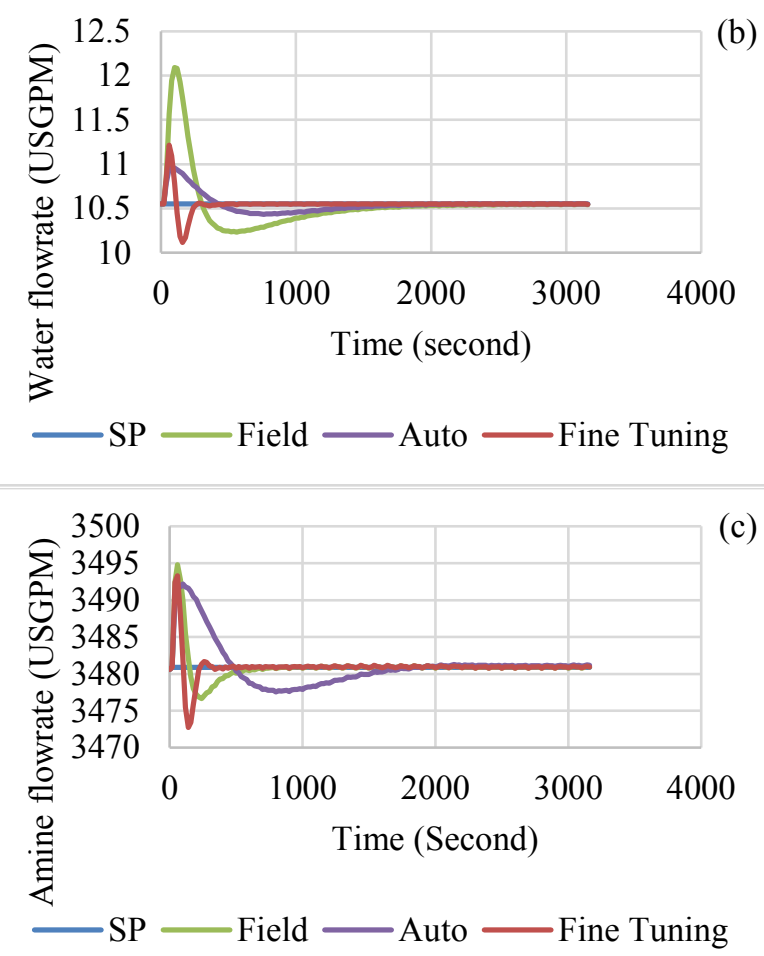

Fig. 5. Disturbance Rejection Results: (a) PIC - 1101, (b) FIC -1102 , (c) FIC -1103

Table 5. ISE Value for Disturbance Rejection

\begin{tabular}{|c|c|c|c|}
\hline Controllers & Fine Tuning & Field & Autotuner \\
\hline PIC-1101 & 62.45 & 447.26 & 428.31 \\
\hline
\end{tabular}

\begin{tabular}{|c|c|c|c|}
\hline FIC-1102 & 1.45 & 15.76 & 1.69 \\
\hline FIC-1103 & 524 & 697 & 1564 \\
\hline
\end{tabular}

\section{Conclusion}

The result of this research shows that fine tuning method give the best control performance compared to previous settings in the field, hence can improve controller performance on the field. For $-5 \%$ set point (SP) tracking, retuning with fine tuning gives $77.42 \%$ improvement for PIC - 1101, 90.59\% for FIC - 1102, and $13.06 \%$ for FIC - 1103. While for the disturbance rejection, fine tuning gives $86.04 \%$ improvement for PIC - 1101, 90.8\% for FIC -1102 , and $24.8 \%$ for FIC -1103 . The disadvantage of this study is that some retuning method should be conducted first so fine tuning method could be applied, hence this study takes time.

We express our gratitude to the Universitas Indonesia which has funded this research through the scheme of Hibah Publikasi Internasional Terindeks untuk Tugas Akhir Mahasiswa (PITTA) No.2359/UN2.R3.1/HKP.05.00/2018.

\section{References}

1. M. Veronesi, A. Visioli, Ind and Eng. Chem. Res, 48 , 2616 - 2623 (2009)

2. M. Veronesi, A. Visioli, Proceeding of IFAC Conference on Advances in PID Control, 28 - 30 (2012)

3. M. Veronesi, A. Visioli, ISA Transactions, 49(2), 244 -248 (2010)

4. M. Veronesi, A. Visioli, Processes, 3, $113-137$ (2015)

5. PT X, Process Flow and Balance Diagram for Main Process $\mathrm{CO}_{2}$ Removal (2000)

6. A.C. Smith, A.B. Corripio, Principles and Practice of Automatic Process Control (1997)

7. T.E. Marlin, Process Control (2nd ed) (2000)

8. A. Wahid, R. Gunawan, Proceeding of National Seminar of Chemical Process Technology VII 2005, $1-9(2005)$ 